PROCEEDINGS OF THE

AMERICAN MATHEMATICAL SOCIETY

Volume 133, Number 11, Pages 3163-3169

S 0002-9939(05)07869-X

Article electronically published on May 2, 2005

\title{
REMARKS CONCERNING LINEAR CHARACTERS OF REFLECTION GROUPS
}

\author{
G. I. LEHRER
}

(Communicated by John R. Stembridge)

\begin{abstract}
Let $G$ be a finite group generated by unitary reflections in a Hermitian space $V$, and let $\zeta$ be a root of unity. Let $E$ be a subspace of $V$, maximal with respect to the property of being a $\zeta$-eigenspace of an element of $G$, and let $C$ be the parabolic subgroup of elements fixing $E$ pointwise. If $\chi$ is any linear character of $G$, we give a condition for the restriction of $\chi$ to $C$ to be trivial in terms of the invariant theory of $G$, and give a formula for the polynomial $\sum_{x \in G} \chi(x) T^{d(x, \zeta)}$, where $d(x, \zeta)$ is the dimension of the $\zeta$-eigenspace of $x$. Applications include criteria for regularity, and new connections between the invariant theory and the structure of $G$.
\end{abstract}

\section{BACKGROUND AND NOTATION}

In this section we recall some basic and well-known facts concerning reflection groups, most of which may be found in [OT].

1.1. Invariant theory. Let $G$ be a finite group generated by (pseudo)reflections in a complex vector space $V$ of dimension $\ell>0$. For any linear transformation $A: V \longrightarrow V$ and element $\zeta \in \mathbb{C}$, denote by $V(A, \zeta)$ the $\zeta$-eigenspace of $A$, and by $d(A, \zeta)$ its dimension. It is well known that if $S$ denotes the coordinate ring of $V$ (identified with the symmetric algebra on the dual $V^{*}$ ) the $\operatorname{ring} S^{G}$ of polynomial invariants of $G$ is free; if $f_{1}, f_{2}, \ldots, f_{\ell}$ is a set of homogeneous free generators of $S^{G}$, then the degrees $d_{i}=\operatorname{deg} f_{i}(i=1, \ldots, \ell)$ are determined by $G$ and are called the invariant degrees of $G$.

Let $F$ be the ideal of $S$ generated by the elements of $S^{G}$ which vanish at $0 \in V$. The space $S / F:=S_{G}$ is called the coinvariant algebra of $(G, V)$. It is clearly graded, and for each $i$, the graded component $\left(S_{G}\right)_{i}$ of degree $i$ is a $G$-module. By a classical result of Chevalley, $S_{G}$ realises the regular representation of $G$, and we have an isomorphism of $\mathbb{C} G$-modules: $S \stackrel{\sim}{\longrightarrow} S^{G} \otimes_{\mathbb{C}} S_{G}$. We shall identify the $G$-module $S_{G}$ with the $G$-invariant complement $\mathcal{H}_{G}$ of $F$ in $S$ comprising the harmonic polynomials, i.e., those polynomials $f \in S$ which are annihilated by $S(V)^{G}$, regarded as differential operators on $S=S\left(V^{*}\right)$. Then Chevalley's isomorphism may be interpreted as the assertion that $S=S^{G} \otimes \mathcal{H}_{G}$.

For any (finite-dimensional) $\mathbb{C} G$-module $M$, the space

$$
\left(S \otimes M^{*}\right)^{G}=S^{G} \otimes\left(\mathcal{H}_{G} \otimes M^{*}\right)^{G}
$$

Received by the editors December 12, 2003 and, in revised form, June 8, 2004 and June 14, 2004.

2000 Mathematics Subject Classification. Primary 20F55; Secondary 14G05, 20G40, 51F15.

(C)2005 American Mathematical Society 
is clearly free as a module over $S^{G}$, with $S^{G}$-basis a linear basis of $\left(\mathcal{H}_{G} \otimes M^{*}\right)^{G}$. Let $\left\{u_{1}, \ldots, u_{r}\right\}(r=\operatorname{dim} M)$ be such a basis. The $u_{i}$ may be taken to be homogeneous in $\mathcal{H}_{G}$, and the degrees $m_{1}(M) \leq m_{2}(M) \leq \cdots \leq m_{r}(M)$ of the $u_{i}$, where $r=$ $\operatorname{dim} M$, are called the $M$-exponents of $G$. They are independent of the basis. The $V$-exponents are the usual exponents $\left\{m_{1}, \ldots, m_{\ell}\right\}$ of $G$, while the $V^{*}$-exponents $\left\{m_{1}^{*}, \ldots, m_{\ell}^{*}\right\}$ are usually referred to as the co-exponents of $G$.

The associative algebra $\left(S \otimes \Lambda M^{*}\right)^{G}$ is bigraded (or $\mathbb{Z} \times \mathbb{Z}$-graded), where we take the usual grading on $S$, and declare the elements of $M^{*}$ to have degree 1 , so that if $f$ is a homogeneous element of degree $a$ in $S$, the (bi)degree of $f \otimes y_{1} \wedge y_{2} \wedge \cdots \wedge y_{b}$ is $(a, b)$. Moreover, since the elements of fixed degree $(a, b)$ form a finite-dimensional subspace $\left(S \otimes \Lambda M^{*}\right)_{(a, b)}^{G}$, we may define the Poincaré series

$$
P_{\left(S \otimes \Lambda M^{*}\right)^{G}}(x, y):=\sum_{(a, b) \in \mathbb{Z} \times \mathbb{Z}} \operatorname{dim}\left(S \otimes \Lambda M^{*}\right)_{(a, b)}^{G} x^{a} y^{b} .
$$

We shall use similar notation for other related bigraded spaces.

Now let $\chi$ be a linear character of $G$, i.e., a group homomorphism $G \rightarrow \mathbb{C}^{\times}$. For any $\mathbb{C} G$-module $U$, the element $e \in U$ is said to be $\chi$-invariant if $g e=\chi(g) e$ for each $g \in G$. The subspace of $\chi$-invariant elements of $U$ is denoted by $U^{\chi}$. If $\mathbb{C}_{\chi}$ is the 1-dimensional $G$-module affording $\chi$, there are canonical isomorphisms

$$
U^{\chi} \stackrel{\sim}{\longrightarrow} \operatorname{Hom}_{G}\left(\mathbb{C}_{\chi}, U\right) \stackrel{\sim}{\longrightarrow}\left(U \otimes \mathbb{C}_{\chi}^{*}\right)^{G} .
$$

Applying (1.2) to the case $U=S$, it follows from Chevalley's result above that there is a homogeneous polynomial $\Pi_{\chi} \in \mathcal{H}_{G}$, unique up to multiplication by a non-zero scalar, such that $S^{\chi}=\Pi_{\chi} S^{G}$. The unique $\mathbb{C}_{\chi}$-exponent $m(\chi)$ is simply the degree of $\Pi_{\chi}$. When $\chi=\operatorname{det}_{V}=\operatorname{det}$ (the determinant character), $\Pi_{\operatorname{det}}=\Pi$, the unique (up to multiplication by a non-zero scalar) polynomial of highest degree in $\mathcal{H}_{G}$. For any $\chi$ and any $\mathbb{C} G$-module $M$, denote by $M \chi$ the module $M \otimes \mathbb{C}_{\chi}$.

1.2. Eigenspaces. Let $d$ be any positive integer, and let $\zeta \in \mathbb{C}$ be a primitive $d^{\text {th }}$ root of unity. Suppose $g \in G$ is such that $E:=V(g, \zeta)$ is maximal among $\zeta$ eigenspaces of elements of $G$. It was shown in $\mathrm{Sp}$ that this defines $E$ uniquely up to transformation by an element of $G$, and that $\operatorname{dim} E=a(d):=\mid\{i \in\{1, \ldots, \ell\} \mid$ $d$ divides $\left.d_{i}\left(=\operatorname{deg} f_{i}\right)\right\} \mid$. Define subgroups $N=\{x \in G \mid x E \subset E\}$ and $C=\{x \in$ $G \mid x e=e$ for all $e \in E\}$. It is a result of LS1 that the group $N / C$ acts faithfully as a unitary reflection group on $E$.

The integer $d$ is said (cf. $[\mathrm{Sp}$ ) to be regular for $G$ if the maximal eigenspace $E$ contains a vector which is regular for $G$, i.e., which lies in no reflecting hyperplane. Equivalently, $E$ is not contained in any reflecting hyperplane. It follows from Steinberg's theorem ( $[\mathrm{St}, 1.5]$ ) that the integer $d$ is regular for $G$ if and only if $C=\{1\}$.

1.3. Notation. In addition to that which was introduced in the above discussion, we shall make use of the following notation. The set of reflecting hyperplanes of $G$ in $V$ is denoted $\mathcal{A}_{G}$. For $H \in \mathcal{A}_{G}$, the cyclic group $G_{H}$ of reflections in $H$ has order $e_{H}$. If $L_{H} \in S$ denotes a linear form defining $H$, it is known that in the notation above, $\Pi_{\mathrm{det}}=\prod_{H \in \mathcal{A}_{G}} L_{H}^{e_{H}-1}$, and $\Pi_{\mathrm{det}^{-1}}=\prod_{H \in \mathcal{A}_{G}} L_{H}$. Thus we have $m(\operatorname{det})=\sum_{H \in \mathcal{A}_{G}}\left(e_{H}-1\right):=n_{G}$, the number of reflections in $G$ and $m\left(\operatorname{det}^{-1}\right)=\left|\mathcal{A}_{G}\right|:=N_{G}$, the number of reflecting hyperplanes. It is well known 
(cf. Corollary 3.6 below) that

$$
N_{G}=\sum_{i=1}^{\ell} m_{i}^{*}=\left|\mathcal{A}_{G}\right| \text { and } n_{G}=\sum_{i=1}^{\ell} m_{i}=\sum_{H \in \mathcal{A}_{G}}\left(e_{H}-1\right) .
$$

Now fix a linear character $\chi$ and integer $d$ as above. The module $V \chi:=V \otimes \chi$ has exponents which we denote by $m_{i}(V \chi)(i=1, \ldots, \ell)$. Define $r_{i}=r_{i}(\chi):=$ $m(\chi)-m_{i}(V \chi)(i=1, \ldots, \ell)$, and let $c(\chi, d)$ be the integer defined by

$$
c(\chi, d)=\mid\left\{i \in\{1, \ldots, \ell\} \mid d \text { divides } r_{i}-1\right\} \mid .
$$

Consider the special case $\chi=\operatorname{det}^{-1}$ of these definitions. It is clear that $V \otimes$ $\operatorname{det}^{-1} \cong \Lambda^{\ell-1} V^{*}$, whence the exponents are given (cf. [LM] by $m_{j}\left(V \operatorname{det}^{-1}\right)=$ $\sum_{i \neq j} m_{i}^{*}$. Hence $r_{j}=m\left(\operatorname{det}^{-1}\right)-m_{j}\left(V \operatorname{det}^{-1}\right)=N_{G}-m_{j}\left(V \operatorname{det}^{-1}\right)=m_{j}^{*}$ by (1.3). Thus the $r_{j}(\chi)$ are generalisations of the co-exponents.

Another special case is when $\chi=1_{G}$, the trivial character. In this case $m(\chi)=0$ and $r_{j}\left(1_{G}\right)=-m_{j}$.

\section{THE MAIN THEOREM}

Our main result is the following theorem. We shall see shortly (Remark 3.7) that it simultaneously generalises formulae of Pianzola-Weiss (the case $\chi=1_{G}$ ), and Lehrer-Michel (the case $\chi=\operatorname{det}^{-1}$ ).

Theorem 2.1. Fix an integer $d$ and character $\chi$ of the reflection group G. With notation as in $\S 1$, assume the invariant degrees $d_{i}$ are ordered so that $d \mid d_{i} \Longleftrightarrow$ $1 \leq i \leq a(d)$. Then

(i) We have $a(d) \leq c(\chi, d)$, so we may order the $r_{j}(\chi)$ so that $d \mid r_{j}-1$ for $1 \leq j \leq a(d)$.

(ii) The following polynomial identity is valid in $\mathbb{C}[T]$ :

$$
\sum_{g \in G} \chi(g) T^{d(g, \zeta)}=\zeta^{-m(\chi)} \prod_{j \leq a(d)}\left(T-r_{j}(\chi)\right) \prod_{j>a(d)} d_{j}\left(\frac{1-\zeta^{r_{j}-1}}{1-\zeta^{-d_{j}}}\right) .
$$

Proof. This will be similar to the proofs of the results in $[\mathrm{LM}]$. We first prove an identity in rational functions of two variables, which is similar to results in [OS, LM], and then compute the residue at a certain pole after an appropriate substitution of the variables. The identity is

$$
|G|^{-1} \sum_{g \in G} \frac{\operatorname{det}_{V}(1+y g)}{\operatorname{det}_{V}(1-x g)} \chi(g)=x^{m(\chi)} \frac{\prod_{j=1}^{\ell}\left(1+y x^{m_{j}(V \chi)-m(\chi)}\right)}{\prod_{i=1}^{\ell}\left(1-x^{d_{i}}\right)} .
$$

For the proof of (2.3) we begin by recalling the following result, which was proved in [Sh, Cor. 1, Prop. 2, Cor. 2].

2.4. In the above notation, let $\Omega=S \otimes \Lambda V^{*}=\bigoplus_{p=0}^{\ell} S \otimes \Lambda^{p} V^{*}$, and let $\chi$ be a linear character of the reflection group $G$.

(i) If $\omega, \omega^{\prime}$ are homogeneous elements of $\Omega^{\chi}$, then $\Pi_{\chi}$ divides $\omega \wedge \omega^{\prime}$. Thus $\omega \curlywedge \omega^{\prime}:=\Pi_{\chi}^{-1} \omega \wedge \omega^{\prime}$ is in $\Omega^{\chi}$.

(ii) The space $\left(S \otimes V^{*}\right)^{\chi}$ is free as an $S^{G}$-module. If $\left\{\omega_{1}, \ldots, \omega_{\ell}\right\}$ is a basis, then for $1 \leq p \leq \ell,\left(S \otimes \Lambda^{p} V^{*}\right)^{\chi}$ has $S^{G}$-basis $\left\{\omega_{i_{1}} \curlywedge \omega_{i_{2}} \curlywedge \cdots \curlywedge \omega_{i_{p}} \mid 1 \leq\right.$ $\left.i_{1}<i_{2}<\cdots<i_{p} \leq \ell\right\}$. 
We shall now compute the Poincaré series (cf. (1.1)) of the bigraded space $\left(S \otimes \Lambda V^{*}\right)^{\chi}$ in two different ways. First, by an obvious extension of Molien's formula, if $M=\bigoplus M_{(a, b)}$ is bigraded, with $\operatorname{dim} M_{(a, b)}<\infty$, then

$$
P_{M \chi}(x, y)=|G|^{-1} \sum_{g \in G} \operatorname{Trace}\left(g, M_{(a, b)}\right) \overline{\chi(g)} x^{a} y^{b} .
$$

Applying this general formula to the case $M=S \otimes \Lambda V^{*}$, we obtain the complex conjugate of the left side of (2.3). It will suffice to prove that this is equal to the right side, since the latter is clearly invariant under complex conjugation.

For the right side, let $W$ be the vector space with basis $\left\{\omega_{1}, \ldots, \omega_{\ell}\right\}$ in the notation of 2.4(ii), and let $\curlywedge W$ be the algebra generated by $W$ under the $\curlywedge$ operation, where $\iota^{0} W$ is interpreted as $\mathbb{C} \Pi_{\chi}$. Then 2.4(ii) asserts that

$$
\left(S \otimes \Lambda V^{*}\right)^{\chi} \cong S^{G} \otimes \curlywedge W,
$$

whence

$$
P_{\left(S \otimes \Lambda V^{*}\right) \chi}(x, y)=P_{S^{G}}(x) P_{\curlywedge W}(x, y) .
$$

To compute the Poincaré series, observe that the $\curlywedge$ operation on $W$ is additive in the shifted bidegree $\delta(f \otimes \lambda):=(\operatorname{deg}(f)-m(\chi), \operatorname{deg}(\lambda))\left(f \in S, \lambda \in \Lambda V^{*}\right)$. Moreover the shifted bidegrees $\delta\left(\omega_{i}\right)=\left(m_{i}(V \chi)-m(\chi), 1\right)=\left(-r_{i}, 1\right)$ for $i=1,2, \ldots, \ell$ in some order. It follows that

$$
P_{\curlywedge W}(x, y)=x^{m(\chi)} \prod_{i=1}^{\ell}\left(1+y x^{-r_{i}}\right),
$$

and the formula (2.3) follows from (2.5).

Now make the substitution $1+\zeta y=T(1-\zeta x)$, i.e. $y=\zeta^{-1}(T(1-\zeta x)-1)$ in (2.3). Then the term corresponding to each $g \in G$ on the left side is analytic at $x=$ $\zeta^{-1}$ (for any $T$ ), and hence we may put $x=\zeta^{-1}$, obtaining $|G|^{-1} \sum_{g \in G} T^{d(g, \zeta)} \chi(g)$.

Making the same substitution on the right side, since the denominator has a zero of order $a(d)$ at $x=\zeta^{-1}$ and the left side is analytic, it follows that the numerator has a zero of order at least $a(d)$; but it is easily checked that each factor $\left(1+y x^{-r_{i}}\right)$ has at most a simple zero at $x=\zeta^{-1}$, and this happens precisely when $d \mid r_{i}-1$. Thus $a(d) \leq c(d, \chi)$ and if we have equality, one computes the limit as $x \longrightarrow \zeta^{-1}$ of the right side using de l'Hôspital's rule. This completes the proof of the theorem.

\section{Applications}

3.1. Special cases of the basic equation. The first case we record is simply when $d=\zeta=1$ in (2.2), which is sometimes useful for computation of the $r_{i}(\chi)$.

Corollary 3.1. For any linear character $\chi$ of $G$, we have

$$
\sum_{g \in G} \chi(g) T^{d(g, 1)}=\prod_{j=1}^{\ell}\left(T-r_{j}(\chi)\right),
$$

where $r_{j}(\chi)=m(\chi)-m_{j}(V \chi)$. 
This leads to

Corollary 3.3. (i) The multisets $\left\{r_{1}(\chi), \ldots, r_{\ell}(\chi)\right\}$ and $\left\{r_{1}(\bar{\chi}), \ldots, r_{\ell}(\bar{\chi})\right\}$ are equal.

(ii) Say that $g \in G$ is elliptic if $g$ has no fixed points on $V$. Then

$$
\sum_{\substack{g \in G \\ \text { elliptic }}} \chi(g)=(-1)^{\ell} \prod_{i=1}^{\ell} r_{i}(\chi) .
$$

(iii) We have

$$
\sum_{i=1}^{\ell} r_{i}(\chi)=\left|\mathcal{A}_{G}\right|-\sum_{\substack{\left.H \in \mathcal{A}_{G} \\ \chi\right|_{G}=1_{G}}} e_{H}
$$

Proof. Take the complex conjugate of both sides in (3.2). The right side is unchanged, while the left side equals $\sum_{g \in G} \bar{\chi}(g) T^{d(g, 1)}$, since $d(g, 1)=d\left(g^{-1}, 1\right)$. The statement (i) now follows by applying (3.2) with $\bar{\chi}$ replacing $\chi$. The equation in (ii) arises by comparing the constant terms of the two sides of (3.2). For (iii) consider the coefficient of $T^{\ell-1}$ on both sides of (3.2). The elements $g$ with $d(g, 1)=\ell-1$ are just the reflections, and $\sum_{g: V(g, 1)=H} \chi(g)$ is -1 or $e_{H}-1$, according as the restriction to $G_{H}$ of $\chi$ is non-trivial or not. The equation (iii) follows.

Corollary 3.6. (i) We have $r_{i}(\operatorname{det})=r_{i}\left(\operatorname{det}^{-1}\right)=m_{i}^{*}$, for some numbering of the co-exponents.

(ii) The $V \otimes$ det-exponents are $\left\{n_{G}-m_{j}^{*}, j=1, \ldots, \ell\right\}$.

Proof. We have seen in subsection 1.3 above that the multiset $\left\{r_{i}\left(\operatorname{det}^{-1}\right)\right\}$ is equal to $\left\{m_{i}^{*}\right\}$. Hence (i) is immediate from 3.3 (i). For (ii), observe that by (i), for some numbering of the co-exponents, $r_{i}(\operatorname{det})=m_{i}^{*}=n_{G}-m_{i}(V$ det $)$.

Remark 3.7. The statement 3.3(i) is Shepler's [Sh04 Cor. 13(g)], and 3.6 is a special case of that result. Also, our Corollary 3.3(iii) generalises [Sh04, Cor. 13(e)].

When $\chi=1_{G}, r_{i}(\chi)=-m_{i}$ by the remark in subsection 1.3. Hence (3.5) asserts that $\sum_{i} m_{i}=n_{G}$, while if $\chi=\operatorname{det}^{-1}$, then by [3.6(i), (3.5) asserts that $\sum_{i} m_{i}^{*}=N_{G}$. Thus (3.5) generalises the well-known relation (1.3). Similarly, it is well known that the number of elliptic elements is $\prod_{i} m_{i}$, which is the case $\chi=1_{G}$ of (3.4).

In view of $3.6(\mathrm{i})$, the main result $(2.2)$ generalises ([LM, Proposition 2.9]), which is just the case $\chi=\operatorname{det}^{-1}$ of (2.2), and which was used in loc. cit. to show that $d$ is regular for $G$ precisely when $d$ divides the same number of degrees as codegrees (see [3.10 below). Similarly, the case $\chi=1_{G}$ of (2.2) is the equation of Pianzola and Weiss [PW].

\subsection{Applications to the theory of eigenspaces of elements of $G$.}

Corollary 3.8. Let $d, \zeta, E, N$ and $C$ be as in $\S 1$ above. Let $\chi$ be a linear character of $G$. Then the following are equivalent.

(i) The restriction $\operatorname{Res}_{C}^{G}(\chi)$ is non-trivial.

(ii) In the notation of Theorem 2.1, $c(\chi, d)>a(d)$.

(iii) $\sum_{g \in G} \chi(g) T^{d(g, \zeta)}=0$. 
Proof. Assume (i); then the leading coefficient (i.e. the coefficient of $T^{a(d)}$ of the left side of Theorem 2.1(ii)) is zero, since $\{g \in G \mid d(g, \zeta)=a(d)\}$ is a disjoint union of cosets of conjugates of the parabolic subgroup $C$, because $\{g \in G \mid V(g, \zeta)=$ $E\}=h C$ for some element $h \in G$. Comparing with the right side of Theorem 2.1(ii), we see that $c(\chi, d)>a(d)$, whence (ii). Trivially (ii) implies (iii), and (iii) implies (i) by the reverse of the argument just given.

Corollary 3.9. (i) If $d$ is regular for $G$, then $a(d)=c(\chi, d)$ for any linear character $\chi$.

(ii) If the restriction of $\chi$ to the isotropy group $G_{H}$ of each reflecting hyperplane $H$ of $G$ is non-trivial, the converse of (i) holds, i.e. if $a(d)=c(\chi, d)$, then $d$ is regular.

Proof. The statement 3.9) $\mathrm{i}$ ) is clear from Corollary 3.8 because $d$ is regular precisely when $C=\{1\}$, in which case the restriction of $\chi$ to $C$ is obviously trivial. To see (ii), observe that if $a(d)=c(\chi, d)$, then by inspection of the right side of the equation in Theorem 2.1(ii), the coefficient of $T^{a(d)}$ in $\sum_{g \in G} \chi(g) T^{d(g, \zeta)}$ is nonzero. But this coefficient on the left side has a factor $\sum_{x \in C} \chi(x)$. Moreover if the parabolic subgroup $C$ is non-trivial, it contains some isotropy subgroup $G_{H}$, whence the restriction to $C$ of $\chi$ is non-trivial, and so $\sum_{x \in C} \chi(x)=0$. It follows that if $a(d)=c(\chi, d)$, then $C$ is trivial, and so $d$ is regular.

Remark 3.10. We have seen that when $\chi=\operatorname{det}^{-1}$, then $c(\chi, d)=b(d)$, the number of codegrees which are divisible by $d$. Hence 3.9 generalises the result of Lehrer and Springer (cf. [LS2, LM]).

Note that the condition in 3.9 (ii) is necessary. For example if $\chi=1_{G}$, the trivial character of $G$, we have seen in subsection 1.3 that $r_{i}=-m_{i}$, whence $c(\chi, d)$ is $\mid\left\{i \mid d\right.$ divides $\left.-\left(m_{i}+1\right)\right\} \mid$, which is equal to $a(d)$ for any $d$.

Corollary 3.11. Suppose $a(d)=c(\chi, d)$, and that $g \in G$ has $\zeta$-eigenspace of maximal dimension $a(d)$. Then

$$
\chi(g)=\zeta^{-m(\chi)} \prod_{j>a(d)}\left(\frac{1-\zeta^{r_{j}-1}}{1-\zeta^{-d_{j}}}\right) .
$$

Proof. Let $E$ be a maximal $\zeta$-eigenspace. The set $\{g \in G \mid V(g, \zeta)=E\}$ is a coset $h C$. Although the elements of $h C$ may not all be conjugate in $G$, since $\chi$ restricts trivially to $C$ by Corollary 3.8 $\chi$ takes the same value on all elements of $h C$, and similarly on all elements $g \in G$ with $d(g, \zeta)=a(d)$. The number of these elements is $|C|$ times the number of maximal $\zeta$-eigenspaces, i.e.,

$$
|C| \times \frac{|G|}{|N|}=\prod_{j>a(d)} d_{j}
$$

The statement now follows from Theorem 2.1(ii).

Remark 3.12. When $d$ is regular, the formula in 3.11 applies for any $\chi$. Moreover it is a result of Springer $[\mathrm{Sp}$ that the eigenvalues of $g$ on any $G$-module $M$ are $\left\{\zeta^{-m_{i}(M)}\right\}$. Applying this respectively to the modules $V, \mathbb{C}_{\chi}$ and $V \chi$, one sees easily that in this case the multisets $\left\{-r_{i}+1\right\}$ and $\left\{d_{i}\right\}(i=1, \ldots, \ell)$ are equal modulo $d$. Hence when $d$ is regular we simply recover from 3.11 the well-known fact that $\chi(g)=\zeta^{-m(\chi)}$. 
We finish with an example.

Example 3.13. Let $G$ be a Coxeter group of type $B_{\ell}$. Let $\delta$ be the character of $G$ whose kernel is the Coxeter group of type $D_{\ell}$ in $G$, and let $\chi=\delta \otimes \operatorname{det}$. If $H \in \mathcal{A}_{G}$ is a reflecting hyperplane, one verifies easily that $\left.\chi\right|_{G_{H}}=1_{G_{H}}$ for just the coordinate hyperplanes $X_{i}=0$ (in the usual notation). Take $d=4$ and $\zeta=\sqrt{-1}$. Then $d$ is not regular if $\ell$ is odd. In this case ( $\ell$ odd), if $E$ is a maximal $\zeta$-eigenspace, there is a unique coordinate hyperplane $H$ containing $E$, and $\left.\chi\right|_{G_{H}}=1$, whence we deduce from Corollary 3.8 that $c(\chi, 4)=a(4)=\frac{\ell-1}{2}$. A short computation using Corollary 3.1. applied to groups $G$ of type $B_{\ell}$ and $D_{\ell}$, shows that the multiset $r_{i}(\chi)$ is equal to $\{1,3, \ldots, 2 \ell-3,-1\}$. One deduces easily using (2.2) that in this case

$$
\sum_{g \in G} \chi(g) T^{d(g, \sqrt{-1})}=(-1)^{\left(\begin{array}{l}
\ell \\
2
\end{array}\right)} \prod_{j=1}^{\frac{\ell-1}{2}}(T-(4 j-3)) \prod_{k=1}^{\frac{\ell-1}{2}}(4 k-2) .
$$

\section{REFERENCES}

[Gu] E.A. Gutkin, "Matrices connected with groups generated by reflections", Funkcional Anal. Appl. 7 (1973), 153-154; translated from Funktsional Anal. i Prilozhen 7 (1973), 81-82. MR.0314956 (47:3505)

[Le] G.I. Lehrer, "Rational tori, semisimple orbits and the topology of hyperplane complements", Comment. Math. Helv. 67 (1992), 226-251. MR1161283 (93e:20065)

[LM] G.I. Lehrer and J. Michel, "Invariant theory and eigenspaces for unitary reflection groups." C. R. Acad. Sc. Paris, Ser. I 336 (2003), 795-800. MR1990017 (2004d:13005)

[LS1] G.I. Lehrer and T.A. Springer, "Intersection multiplicities and reflection subquotients of unitary reflection groups I", Geometric group theory down under (Canberra, 1996), 181193, de Gruyter, Berlin, 1999. MR1714845|(2000i:20064)

[LS2] G.I. Lehrer and T.A. Springer, "Reflection subquotients of unitary reflection groups." Canad. J. Math. 51 (1999), 1175-1193. MR1756877 (2001f:20082)

[OS] P. Orlik and L. Solomon, "Unitary reflection groups and cohomology", Inv. Math. 59 (1980), 77-94. MR0575083 (81f:32017)

[OT] P. Orlik and H. Terao, "Arrangements of hyperplanes." Grundlehren der Mathematischen Wissenschaften, 300, Springer-Verlag, Berlin, 1992. MR.1217488 (94e:52014)

[PW] A. Pianzola and A. Weiss, "Monstrous $E_{10}$ 's and a generalization of a theorem of L. Solomon", C. R. Math. Rep. Acad. Sci. Canada 11 (1989), 189-194. MR1010926 (90k:22007)

[Sh] Anne V. Shepler, "Semi-invariants of finite reflection groups", J. Alg. 220, (1999), 314-326. MR,1714136 (2000g:20069)

[Sh04] Anne V. Shepler, "Generalized exponents and forms", to appear, J. Alg. Comb.

[Sp] T. Springer, "Regular elements of finite reflection groups", Invent. Math. 25 (1974), 159198. MR0354894 (50:7371)

[St] R. Steinberg, "Differential equations invariant under finite reflection groups", Trans. Amer. Math. Soc. 112 (1964), 392-400. MR0167535 (29:4807)

School of Mathematics and Statistics, University of Sydney, New South Wales 2006, Australia

E-mail address: gusl@maths.usyd.edu.au 